\title{
Artigo Análise do Método Loop Flow, Desenvolvido por
Kersting, para Fechamento de Malha entre Duas Fontes
Desequilibradas
}

Márcia Gabriela Leite Dias, ${ }^{1}$ Adriano Aron Freitas de Moura ${ }^{2}$, Ailson Pereira de Moura ${ }^{3}$, Ednardo Pereira da Rocha ${ }^{4}$, Alana Kelly Xavier Santos Campos ${ }^{5}$, Victor de Paula Brandão Aguiar ${ }^{6}$,

${ }^{[1]}$ Universidade Federal Rural do Semi-Árido (UFERSA); adrianoaron@hotmail.com

${ }^{[2]}$ Universidade Federal Rural do Semi-Árido (UFERSA)

${ }^{[3]}$ Universidade Federal do Ceará (UFC)

${ }^{[4]}$ Universidade Federal Rural do Semi-Árido (UFERSA)

${ }^{[5]}$ Universidade Federal Rural do Semi-Árido (UFERSA)

${ }^{[6]}$ Universidade Federal Rural do Semi-Árido (UFERSA)

Recebido: $23 / 04 / 2020$;

Aceito: $30 / 07 / 2020$;

Publicado: 11/09/2020.

Resumo: Através do fluxo de carga em um sistema elétrico de potência pode-se determinar o estado da rede, a forma como os fluxos estão distribuídos e outras grandezas como tensão e corrente. Os métodos tradicionais de cálculo do fluxo de carga, como os métodos de Newton-Raphson e Gauss-Seidel, apresentam problemas de desempenho e convergência devido às características peculiares dos sistemas de distribuição, assim se fez necessário adaptações de métodos iterativos, já utilizados, a fim de corrigir imperfeições técnicas, fazendo com que a caracterização do sistema elétrico de potência seja a mais fidedigna possível. Este trabalho possui o objetivo de analisar um método iterativo, utilizado para análise de fluxo de carga em sistemas de distribuição, apresentado em 2017 por Kersting, denominado Loop Flow. Na apresentação do método por Kersting, são consideradas duas fontes equilibradas para fechar a malha. Entretanto, neste trabalho analisouse o comportamento do método quando se utiliza duas fontes desequilibradas. Para esta situação, o método não convergiu. Dessa forma, adaptou-se o método para que o resultado de convergência fosse obtido.

Palavras-chaves: Fluxo de carga. Sistemas de distribuição. Método Loop Flow.

Abstract: Through the load flow in an electrical power system one can determine the state of the network, how the flows are distributed and other quantities such as voltage and current. The traditional methods of calculating the load flow, such as the Newton-Raphson and Gauss-Seidel methods, present performance and convergence problems due to the peculiar characteristics of the distribution systems, thus it was necessary to adapt iterative methods, already used, in order to correct technical imperfections, making the characterization of the electrical power system as reliable as possible. This work aims to analyze an iterative method, used for load flow analysis in distribution systems, presented in 2017 by Kersting, called Loop Flow. In the presentation of the method, two balanced sources are considered to close the mesh, however in this work it was analyzed that the behavior of the method when using two unbalanced sources. For this situation the result was non-convergence, so the method was adapted so that the convergence result could be obtained.

Key-words: Power flow. Distribution Systems. Loop Flow Method. 


\section{INTRODUÇÃO}

$\mathrm{O}$ estudo do fluxo de potência auxilia em diversos quesitos como: Planejamento, uma vez que utiliza-se o fluxo de potência para simular o funcionamento do sistema elétrico, em caso de expansão para atender novas cargas, melhoramento de sistema já em operação. Através do estudo do fluxo de potência, determina-se pontos de maiores perdas, fazendo com que ações corretivas sejam tomadas, melhorando assim a eficiência. Existe uma gama de trabalhos de análise de fluxo de potência nos setores de geração, transmissão e distribuição de energia elétrica, entretanto vem sendo dada uma atenção cada vez maior aos sistemas de distribuição, pois é o setor que possui as maiores perdas e também é o mais vulnerável às falhas [1].

Os métodos iterativos para análise de fluxo de potência, são abordados na transmissão de energia em alta, extra alta e ultra alta tensão. Exemplos desses métodos são: Gauss-Seidel [2], Newton-Raphson [2] e outros derivados desses. No entanto, quando se tratando de sistemas de distribuição de energia, os métodos citados acima não se aplicam, devido ao grande número necessário de iterações numéricas, o que torna os métodos extremamente lentos e também não precisos, fornecendo resultados que não refletem a realidade com precisão, conforme Kersting em [3] ao falar: "Programas usados na modelagem de sistemas de distribuição fazem a pressuposição que o sistema é equilibrado. Isso torna possível a utilização do equivalente monofásico do sistema. Este não é o caso quando se modela um sistema de distribuição. A natureza desequilibrada do sistema tem que ser modelada." e ressaltar a importância de uma modelagem trifásica dos sistemas de distribuição. Isso ocorre devido aos métodos serem pensados para sistemas de transmissão, nos quais não se considera a estrutura trifásica e os possíveis desequilíbrios entre fases, isto é desconsiderado uma vez que se tem a simetria atingida entre as fases quando em regime permanente. Em contra partida, na análise dos sistemas de distribuição não se pode desconsiderar o desequilíbrio entre as fases, uma vez que o sistema terminal alimenta inúmeras cargas com características diferentes, conectadas de formas diversas, com características radiais ou pouco malhadas. Os principais algoritmos para a solução de fluxo de potência, em sistemas de distribuição, são adequados para sistemas radiais ou fracamente malhados. Exemplos desses são: método Backward-Forward Sweep [4], baseado na técnica Ladder, e derivados do método de Newton-Raphson [2]. A seguir será visto o método Loop Flow que permite incorporar malhas na análise do método Backward/Forward Sweep.

O método Loop Flow foi desenvolvido para casos em que o sistema de distribuição não é completamente radial, o que implica na não possibilidade da utilização dos algoritmos supracitados. Nesses casos, em que o sistema possui apenas algumas malhas, a técnica Ladder Backward-Forward Sweep deve ser modificada, resultando assim no método Loop Flow, o qual trabalha com o sistema, predominantemente radial, porém com malhas.

Este trabalho se justifica na necessidade da análise do método Loop Flow, a fim de indicar possíveis limitações do método, em relação aos ângulos das fontes de tensão, determinando assim sua eficácia e confiabilidade, quando aplicado na análise de fluxo de potência, em sistemas de distribuição.

\section{DESENVOLVIMENTO}

O método em análise, divulgado recentemente, no ano de 2017, por William H. Kersting [2], consiste em uma combinação das técnicas: Ladder e varredura Backward-Forward. Considerando que o método é recente, subentende-se a necessidade de explanação do mesmo, para que se tenha a compreensão do método abordado neste trabalho. 


\subsection{Técnica iterativa Ladder com varredura Backward - Forward}

Conforme [5], o método de varredura Backward/Forward, proposto por D. Shimohammadi em 1988 é baseado na técnica Ladder, proposta por W.H. Kersting em 1976. O método de resolução consiste em dois passos básicos: varredura - backward - onde são calculados as correntes ou fluxos de potência nas linhas, iniciando das barras finais em direção à fonte e a varredura forward - que realiza os cálculos das quedas de tensão com as atualizações das correntes ou fluxos de potência, que parte da fonte em direção as barras no final do circuito. Esses passos são repetidos até que se obtenha a convergência do algoritmo. Por possuir boas características de convergência e ser muito robusto tornou-se o principal método de solução e serviu como base para muitos métodos propostos posteriormente. Este método pode ser aplicado também para sistemas fracamente malhados, ou seja, sistemas que apresentam poucas interligações, onde são convertidos em redes radiais, que é o caso do sistema analisado neste trabalho.

\subsection{Método Loop Flow}

Alguns trabalhos foram desenvolvidos antes de Kersting sobre a temática do método Loop Flow. Em [6], uma técnica de quebra dos loops da rede malhada é proposto, transformando assim a rede em uma rede radial. Em [7] uma técnica para tratar sistemas fracamente malhados é proposta baseada na matriz BIBC. Em [8], um método para analisar a rede de distribuição fracamente malhada é proposto, analisando o impacto da modelagem da carga.

O método Loop Flow, desenvolvido por Kersting [3], combina a técnica apresentada no item 2.1 com a inserção de fontes de corrente. Neste tópico tem-se a explanação do método.

Para análise inicial será considerado um sistema monofásico, conforme representado na Figura 1, na qual tem-se dois sistemas monofásicos, operando independentemente, mas com uma chave entre os dois. Ao fechar a chave, os dois sistemas, anteriormente isolados, passam a compor uma única malha. Nessa condição, com a chave fechada, a diferença de tensão $d V_{34}$ passa a ser zero.

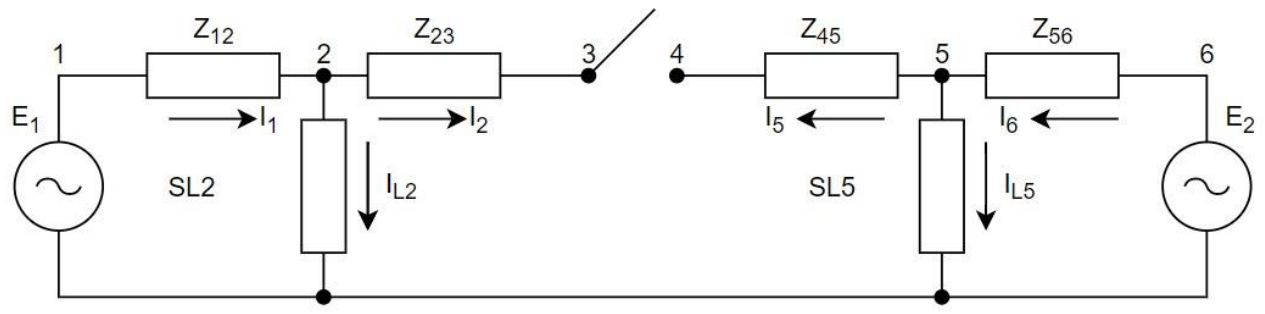

FIGURA 1. Sistema de distribuição monofásico. (Fonte: KERSTING, 2017).

Para que se possa simular o fechamento da chave, ou seja, para que a diferença de tensão entre os nós 3 e 4 seja zero, infere-se que as tensões nos nós devam ser iguais. Na Figura 2 tem-se uma forma de demonstrar que a chave está fechada. Nesta uma corrente IT é injetada no nó 3 e uma corrente - IT é injetada no nó 4. Vale salientar que o valor de IT deve ser determinado de forma correta, para que se garanta $d V_{34}$ igual a zero. 


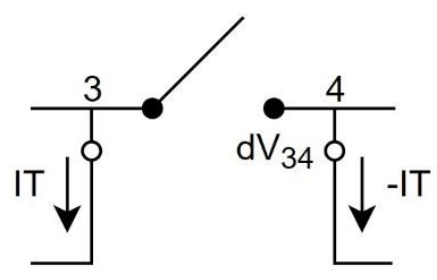

FIGURA 2. Simulação da chave fechada. (Fonte: KERSTING, 2017).

Incluindo as correntes injetadas nos nós 3 e 4, no circuito demonstrado na Figura 1, tem-se a Figura 3.

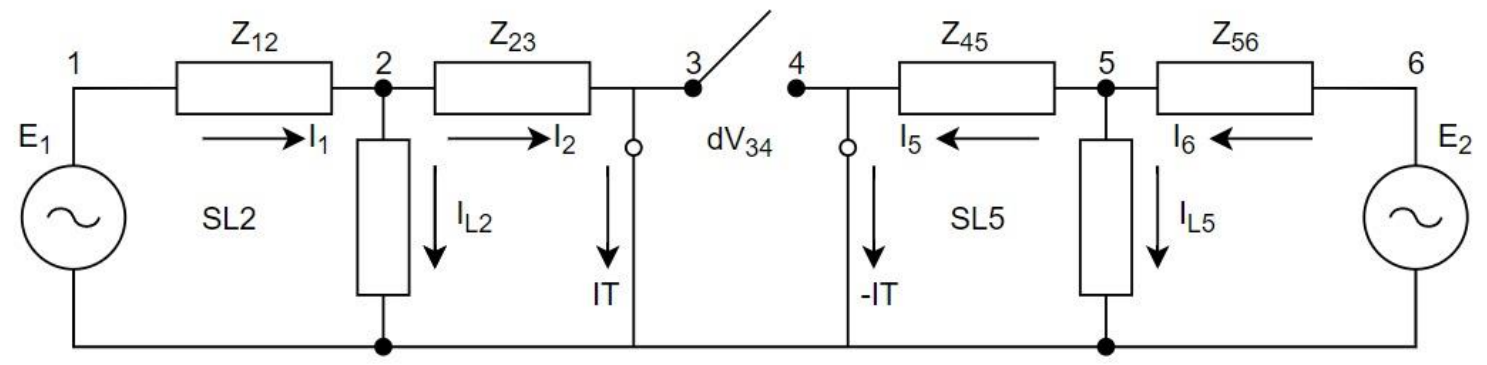

FIGURA 3. Circuito modificado. (Fonte: KERSTING, 2017).

Analisando a Figura 3, tem-se que a tensão no nó 3 é dada por:

$$
\begin{gathered}
\mathrm{V}_{3}=\mathrm{E}_{1}-\mathrm{Z}_{12} \cdot\left(\mathrm{IL}_{2}+\mathrm{IT}\right)-\mathrm{Z}_{23} \cdot \mathrm{IT} \\
\mathrm{V}_{3}=\mathrm{E}_{1}-\mathrm{Z}_{12} \cdot \mathrm{IL}_{2}-\left(\mathrm{Z}_{12}+\mathrm{Z}_{23}\right) \cdot \mathrm{IT}
\end{gathered}
$$

De maneira similar, a tensão no nó 4 pode ser determinada pela análise seguinte:

$$
\begin{gathered}
\mathrm{V}_{4}=\mathrm{E}_{2}-\mathrm{Z}_{56} \cdot\left(\mathrm{IL}_{5}-\mathrm{IT}\right)-\mathrm{Z}_{45} \cdot(-\mathrm{IT}) \\
\mathrm{V}_{4}=\mathrm{E}_{2}-\mathrm{Z}_{56} \cdot \mathrm{IL}_{5}+\left(\mathrm{Z}_{56}+\mathrm{Z}_{45}\right) \cdot \mathrm{IT}
\end{gathered}
$$

A queda de tensão entre os nós 3 e 4 possui uma componente devido às fontes de tensão e outro componente devido às correntes injetadas. Reescrevendo a tensão no nó 3, separando as componentes, tem-se:

$$
\begin{gathered}
\mathrm{V}_{3 \mathrm{~V}}=\mathrm{E}_{1}-\mathrm{Z}_{12} \cdot \mathrm{IL}_{2} \\
\mathrm{~V}_{3 \mathrm{I}}=-\left(\mathrm{Z}_{12}+\mathrm{Z}_{23}\right) \cdot \mathrm{IT} \\
\mathrm{V}_{3}=\mathrm{V}_{3 \mathrm{~V}}+\mathrm{V}_{3 \mathrm{I}}
\end{gathered}
$$

De forma análoga, separando as componentes, da tensão no nó 4, tem-se:

$$
\begin{gathered}
\mathrm{V}_{4 \mathrm{~V}}=\mathrm{E}_{2}-\mathrm{Z}_{56} \cdot \mathrm{IL}_{5} \\
\mathrm{~V}_{4 \mathrm{I}}=\left(\mathrm{Z}_{56}+\mathrm{Z}_{45}\right) \cdot \mathrm{IT} \\
\mathrm{V}_{4}=\mathrm{V}_{4 \mathrm{~V}}+\mathrm{V}_{4 \mathrm{I}}
\end{gathered}
$$

Para que se determine a queda de tensão entre os nós 3 e 4, faz-se a seguinte relação: 


$$
\begin{gathered}
\mathrm{dV}_{34}=\mathrm{V}_{3}-\mathrm{V}_{4} \\
\mathrm{dV}_{34}=\mathrm{V}_{3 \mathrm{~V}}-\mathrm{V}_{4 \mathrm{~V}}+\left(\mathrm{V}_{3 \mathrm{I}}-\mathrm{V}_{4 \mathrm{I}}\right) \\
\mathrm{dV}_{34}=\mathrm{dV}_{34 \mathrm{~V}}-\mathrm{dV}_{34 \mathrm{I}}
\end{gathered}
$$

Utilizando o diferencial de tensão para a componente de injeção de corrente, tem-se:

$$
\begin{gathered}
\mathrm{dV}_{34 \mathrm{I}}=\mathrm{V}_{3 \mathrm{I}}-\mathrm{V}_{4 \mathrm{I}} \\
\mathrm{dV}_{34 \mathrm{I}}=-\left(\mathrm{Z}_{12}+\mathrm{Z}_{23}\right) \cdot \mathrm{IT}-\left(\mathrm{Z}_{56}+\mathrm{Z}_{45}\right) . \mathrm{IT} \\
\mathrm{dV}_{34 \mathrm{I}}=-\left(\mathrm{Z}_{12}+\mathrm{Z}_{23}+\mathrm{Z}_{56}+\mathrm{Z}_{45}\right) . \mathrm{IT}
\end{gathered}
$$

Analisando-se a Equação 5, pode afirmar que a soma das impedâncias corresponde a impedância do equivalente de Thevenin, para o sistema em análise. Para o sistema da Figura 1, determina-se a impedância através do princípio da superposição, no qual as fontes de tensão são curto-circuitadas e somente as correntes injetadas são aplicadas ao circuito, conforme Figura 4.

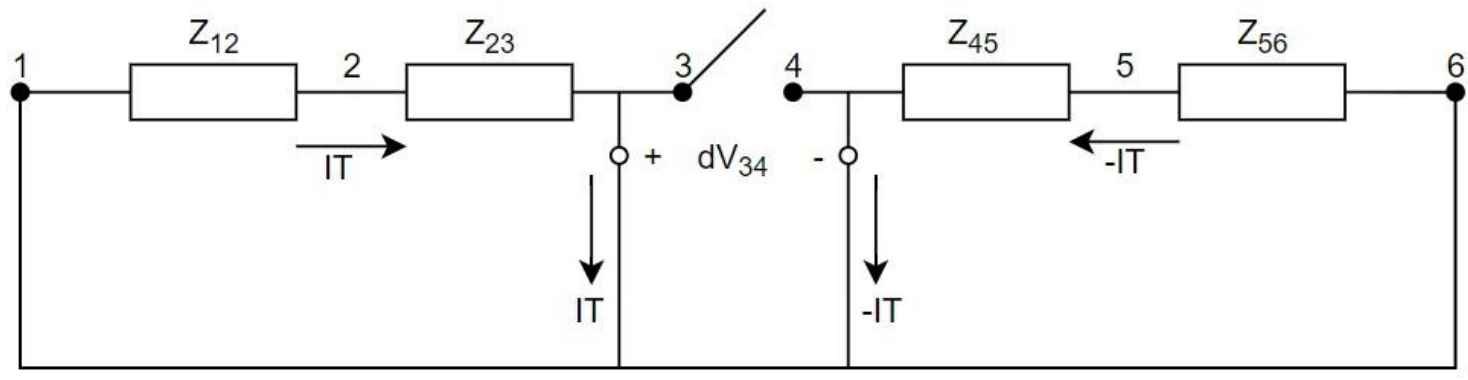

FIGURA 4. Impedância equivalente Thevenin. (Fonte: KERSTING, 2017).

Reescrevendo a Equação 5, tem-se:

$$
\begin{gathered}
\mathrm{dV}_{34 \mathrm{I}}=-\mathrm{Z}_{\text {THEVENIN }} \text { IT } \\
\mathrm{Z}_{\text {THEVENIN }}=-\frac{\mathrm{dV}_{34 \mathrm{I}}}{\mathrm{IT}}
\end{gathered}
$$

Calculada a impedância de Thevenin, deve-se determinar o valor de IT para o qual a diferença de tensão entre os nós 3 e 4 seja zero, simulando assim a chave na posição fechada.

$$
\begin{gathered}
\mathrm{dV}_{34}=\mathrm{dV}_{34 \mathrm{~V}}+\mathrm{dV}_{34 \mathrm{I}}=0 \\
\mathrm{dV}_{34 \mathrm{I}}=-\mathrm{dV}_{34 \mathrm{~V}}
\end{gathered}
$$

Substituindo o valor de $d V_{34 I}$ de acordo com a Equação 6, tem-se:

$$
-\mathrm{Z}_{\text {THEVENIN }} \cdot \mathrm{IT}=-\mathrm{dV}_{34 \mathrm{~V}}
$$

Isolando IT, tem-se:

$$
\mathrm{IT}=\frac{\mathrm{dV}_{34 \mathrm{~V}}}{\mathrm{Z}_{\text {THEVENIN }}}
$$


Como as cargas não são distribuidas linearmente, uma rotina iterativa é utilizada para computar a corrente injetada necessária, para que $d V_{34}$ seja zero. Na Figura 5 tem-se o fluxograma que exemplifica o método Loop Flow.

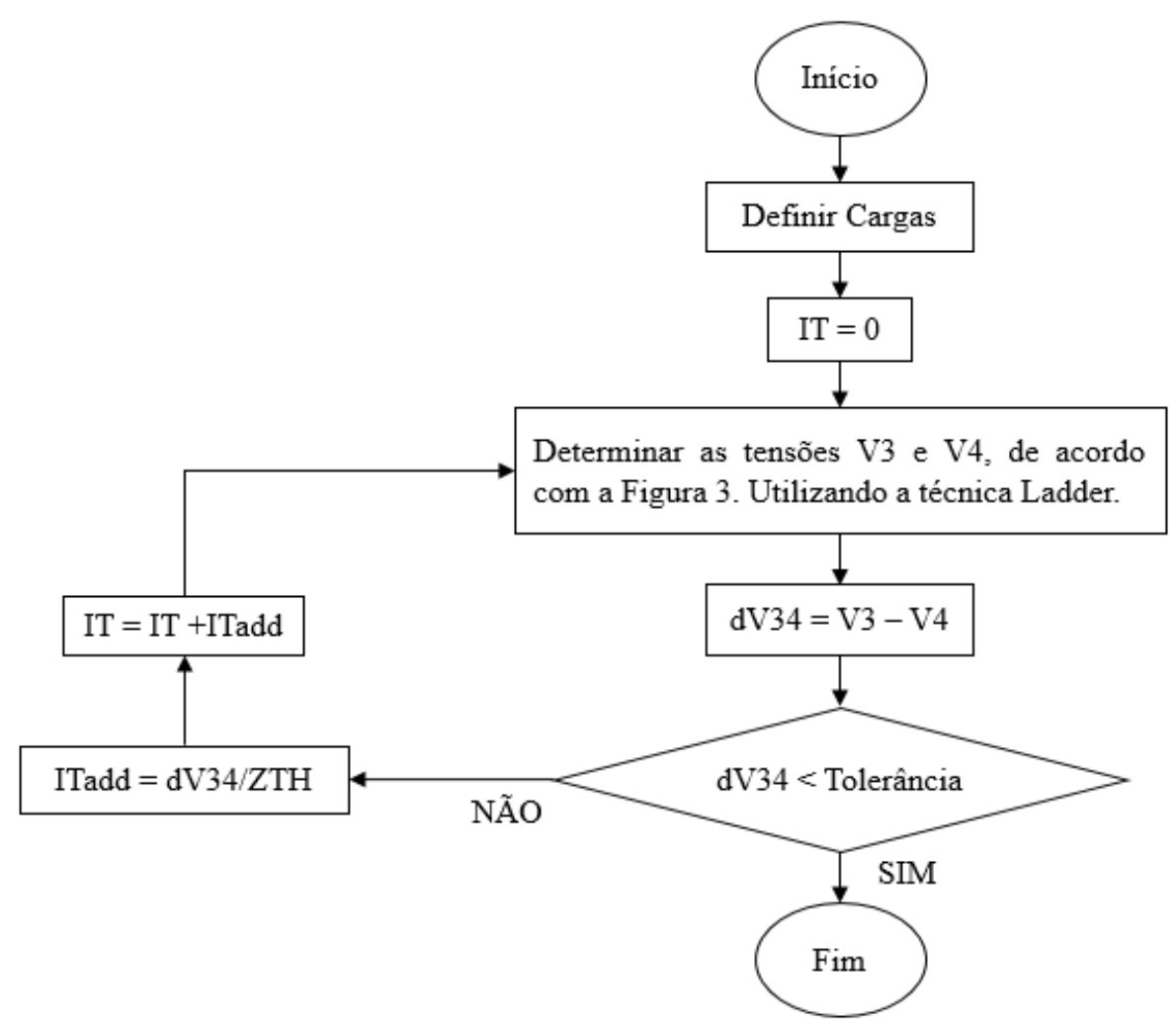

FIGURA 5. Fluxograma para exemplificação da rotina iterativa. (Fonte: Adaptado KERSTING, 2017).

Para esta análise a tolerância será de 0,001 a fim de que se tenha acuracidade nos resultados.

\section{Metodologia}

Para analisar o método Loop Flow implementou-se um algoritmo, de acordo com o fluxograma apresentado na Figura 5, no software MATLAB®. A configuração do sistema para analisar o método está representada na Figura 6, a qual corresponde a um exemplo resolvido, apresentado pelo autor Kersting. Com isso, os valores de impedância e módulos de tensão foram de acordo com o exemplo. Entretanto, como o objetivo do trabalho era analisar a convergência do método, quando utilizando-se duas fontes desequilibradas, acrescentou-se as defasagens das fontes de tensão, que antes eram equilibradas.

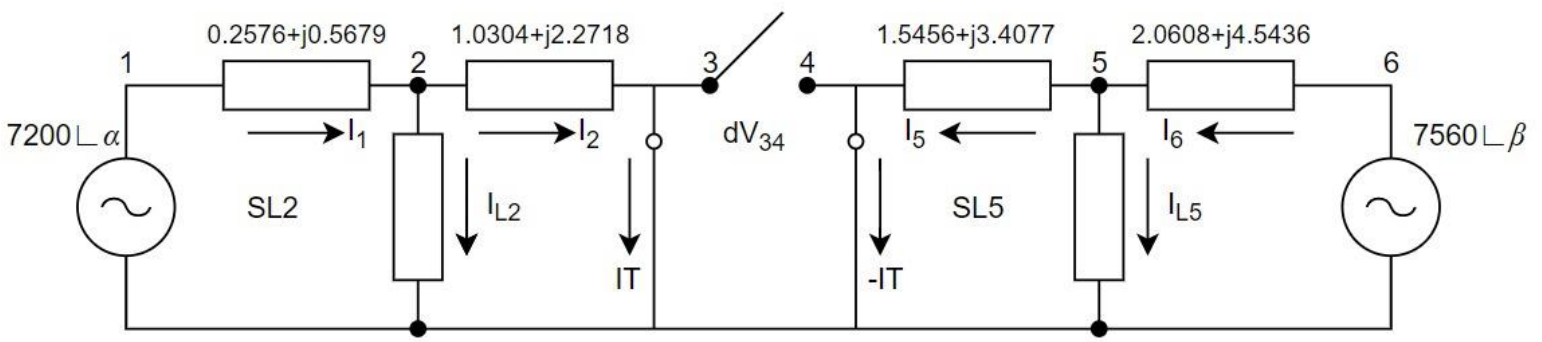

FIGURA 6. Circuito para análise do método. (Fonte: Adaptado KERSTING, 2017).

Os dados do sistema representado na Figura 6, estão expressos abaixo: 


$$
\begin{array}{llc}
Z_{12}=0,2576+j 0,5679 \Omega & \mathrm{SL}_{2}=1500+\mathrm{j} 1250 \mathrm{kVA} & \mathrm{S}_{\text {base }}=1000 \mathrm{kVA} \\
\mathrm{Z}_{23}=1,0304+\mathrm{j} 2,2718 \Omega & \mathrm{SL}_{5}=1000+\mathrm{j} 750 \mathrm{kVA} & \mathrm{V}_{\text {base }}=7200 \mathrm{~V} \\
\mathrm{Z}_{45}=1,5456+\mathrm{j} 3,4077 \Omega & & \mathrm{I}_{\text {base }}=138,98 \mathrm{~A} \\
\mathrm{Z}_{56}=2,0608+\mathrm{j} 4,5436 \Omega & &
\end{array}
$$

Para determinar as correntes IT e -IT utilizou-se o software MATLAB®, para que os cálculos fossem realizados de forma mais rápida e precisa. O código desenvolvido, segue todo o procedimento apresentado na seção 2.1 deste artigo, na Tabela 1 tem-se a representação do mesmo e sua explanação. Nota-se que as fontes

\begin{tabular}{|c|c|}
\hline \multicolumn{2}{|c|}{ Passo 1} \\
\hline $\begin{array}{c}E 1=7200 * \exp (\alpha) \\
E 2=7560 * \exp (\beta) \\
Z 12=0.2576+\mathrm{j} * 0.5679 ; \\
Z 23=1.0304+\mathrm{j} * 2.2718 ; \\
Z 45=1.5456+\mathrm{j} * 3.4077 ; \\
Z 56=2.0608+\mathrm{j} * 4.5436 ;\end{array}$ & $\begin{array}{l}\text { Declaração dos valores de tensão e impedâncias, } \\
\text { fornecidos no esquema do circuito da Figura } 6 \text {. }\end{array}$ \\
\hline \multicolumn{2}{|c|}{ Passo 2} \\
\hline $\begin{array}{l}\text { IT1 }=138.9 \\
\text { IT2=-138.9; } \\
\text { IT }=\text { IT1 }\end{array}$ & $\begin{array}{l}\text { Deseja-se determinar o valor de Zth. Para isso } \\
\text { considera-se a corrente de base como a corrente } \\
\text { injetada (IT) e desconsidera-se as fontes de } \\
\text { tensão. }\end{array}$ \\
\hline $\begin{array}{l}\mathrm{V} 3 \mathrm{I}=-(\mathrm{Z} 12+\mathrm{Z} 23) * \mathrm{IT} \\
\mathrm{V} 4 \mathrm{I}=(\mathrm{Z} 56+\mathrm{Z} 45) * \mathrm{IT}\end{array}$ & $\begin{array}{l}\text { Determinação de V3I e V4I através das } \\
\text { equações de malha. }\end{array}$ \\
\hline \multicolumn{2}{|c|}{ Passo 3} \\
\hline $\begin{array}{l}\mathrm{dV} 34 \mathrm{I}=\mathrm{V} 3 \mathrm{I}-\mathrm{V} 4 \mathrm{I} \\
\mathrm{Zth}=\mathrm{dV} 34 \mathrm{I} / \mathrm{IT} 1\end{array}$ & $\begin{array}{c}\text { Determinação da diferença de tensão devido as } \\
\text { correntes injetadas. } \\
\text { Determinação de Zth. }\end{array}$ \\
\hline \multicolumn{2}{|c|}{ Passo 4} \\
\hline $\mathrm{IT}=0$; & $\begin{array}{c}\text { Deseja-se determinar o valor de V3V e e V4V a } \\
\text { fim de determinar um valor inicial para corrente } \\
\text { injetada. Para determinar a diferença de tensão } \\
\mathrm{dV} 34 \mathrm{~V} \text {, devido às fontes de tensão, no ponto da } \\
\text { chave inserida, desconsidera-se as correntes } \\
\text { injetadas. }\end{array}$ \\
\hline
\end{tabular}
de tensão estão com a defasagem representada por $\alpha$ e $\beta$, as quais durante a simulação, serão variadas de $0^{\circ}$ a $120^{\circ}$.

TABela 1. Código em MATLAB® do método Loop Flow.

Primeira Ladder para determinar V3V e V4V 
$\mathrm{V} 2=7200 * \exp (\alpha)$;

while 1

IL2 $=\operatorname{conj}((1500+\mathrm{j} * 1250) * 1000 / \mathrm{V} 2)$;

$\mathrm{V} 1=\mathrm{V} 2+\mathrm{Z} 12 * \mathrm{IL} 2$;

if $\operatorname{abs}(7200 * \exp (\alpha)-\mathrm{V} 1)<0.001$

break;

end

$\mathrm{V} 2=7200 * \exp (\alpha)-\mathrm{Z} 12 * \mathrm{IL} 2$

end

$\mathrm{V} 3 \mathrm{~V}=7200 * \exp (\alpha)-\mathrm{Z} 12 * \mathrm{IL} 2$;

$\mathrm{V} 5=7560 * \exp (\beta)$

while 1

IL5=conj $((1000+j * 750) * 1000 /$ V5);

$\mathrm{V} 6=\mathrm{V} 5+\mathrm{Z} 56 *$ IL5;

if $\operatorname{abs}(7560 * \exp (\beta)-V 6)<0.001$

break;

end

V5=7560*exp( $\beta)-Z 56 *$ IL5;

end

$\mathrm{V} 4 \mathrm{~V}=7560 * \exp (\beta)-Z 56 *$ IL5;
Nesta etapa utiliza-se a técnica iterativa ladder para determinar $\mathrm{V} 3 \mathrm{~V}$.

A corrente IL2, consumida na carga SL2, tem seu valor determinado de forma que ao ser utilizada na equação da malha, desconsiderando a corrente injetada, forneça um valor de V1 igual ao da fonte $7200 \mathrm{~V}$, sendo aceitável uma tolerância de 0,001 .

A cada iteração é recalculado um valor para V2, que terá influência na determinação do novo valor de IL2.

Determinado o valor aceitável de IL2 determina-se V3V.

Nesta etapa utiliza-se a técnica iterativa ladder para determinar V4V.

A corrente IL5, consumida na carga SL5, tem seu valor determinado de forma que ao ser utilizada na equação da malha, desconsiderando a corrente injetada, forneça um valor de V6 igual ao da fonte $7560 \mathrm{~V}$, sendo aceitável uma tolerância de 0,001 .

A cada iteração é recalculado um valor para V5, que terá influência na determinação do novo valor de IL5.

Determinado o valor aceitável de IL5 determina-se V4V.

Passo 5

Calculados V3V e V4V pode-se determinar a

$\mathrm{dV} 34 \mathrm{~V}=\mathrm{V} 3 \mathrm{~V}-\mathrm{V} 4 \mathrm{~V}$; diferença de tensão dV34V, que junto com o

ITadd $=\mathrm{dV} 34 \mathrm{~V} / \mathrm{Zth}$; valor de Zth, calculado no passo 3, determina

IT $=$ IT+ITadd; um valor de corrente ITadd, que deverá ser adicionado ao valor da corrente injetada, que anteriormente era zero.

Passo 6

Com o novo valor de IT repete-se todo o processo. 
while 1

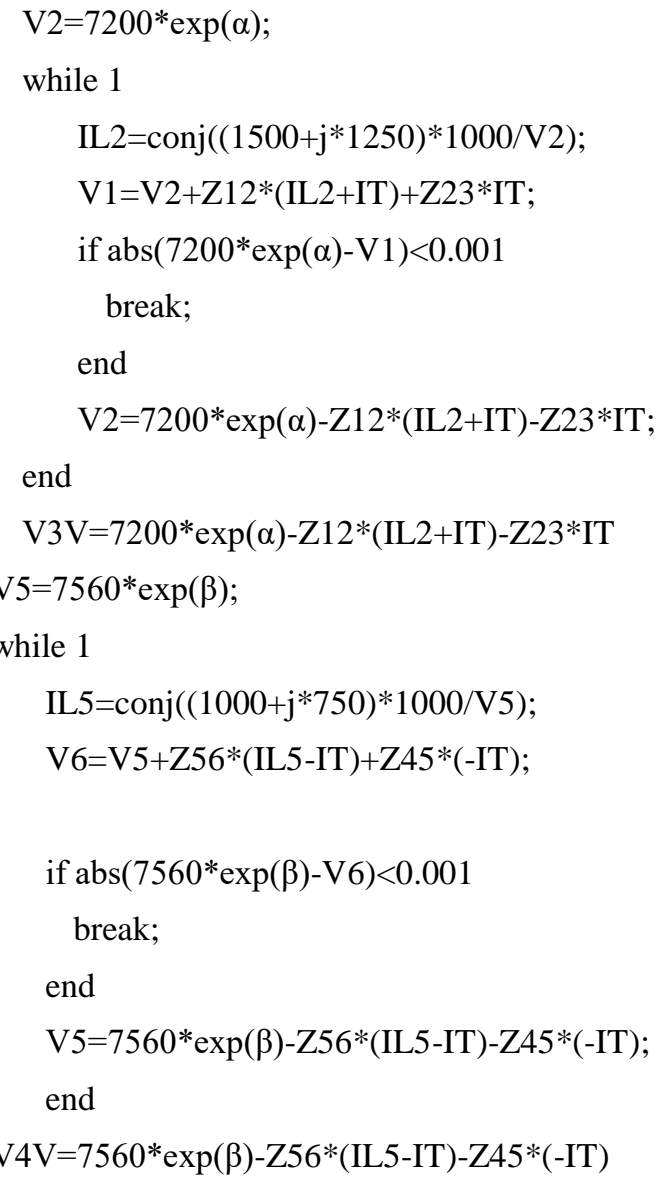

$\mathrm{dV} 34 \mathrm{~V}=\mathrm{V} 3 \mathrm{~V}-\mathrm{V} 4 \mathrm{~V}$;

if $\operatorname{abs}(\mathrm{dV} 34 \mathrm{~V})<0.001$

break;

end

ITadd $=\mathrm{dV} 34 \mathrm{~V} / \mathrm{Zth}$;

IT $=\mathrm{IT}+\mathrm{ITadd}$
Determinação de V3V considerando o valor de

IT.

Determinação de V4V considerando o valor de

IT.

Calculados V3V e V4V pode-se determinar a diferença de tensão dV34V, que deve ser zero. Caso a diferença de tensão seja maior que a tolerância de 0,001 uma nova iteração é feita com um novo valor de ITadd, que será somado ao valor de IT calculado na iteração anterior. Para dV34V menor que 0,001 , significa que o sistema convergiu, determinando valores de IT que satisfazem a condição de tolerância para dV34V.

end Código encerrado.

Fonte: Autoria própria (2018).

\section{RESUltados}

Para discutir a eficácia do método, quando utilizando-se fontes de tensões desequilibradas, simulou-se em MATLAB®, o algoritmo apresentado na Tabela 1, variando os valores das defasagens das fontes de tensão conforme demonstrado na Tabela 2. 
TABELA 2. Variação das defasagens analisadas.

\begin{tabular}{cc}
$E 1$ & $E 2$ \\
$(\mathrm{~V})$ & $(\mathrm{V})$ \\
\hline $7200<\quad 0^{\circ}$ & $7560<00^{\circ}$ \\
$7200<0^{\circ}$ & $7560<120^{\circ}$ \\
$7200<0^{\circ}$ & $7560<-120^{\circ}$ \\
$7200<120^{\circ}$ & $7560<0^{\circ}$ \\
$7200<120^{\circ}$ & $7560<120^{\circ}$ \\
$7200<120^{\circ}$ & $7560<-120^{\circ}$ \\
$7200<-120^{\circ}$ & $7560<0$ \\
$7200<-120^{\circ}$ & $7560<120^{\circ}$ \\
$7200<-120^{\circ}$ & $7560<-120^{\circ}$ \\
\hline
\end{tabular}

Fonte: Autoria própria , 2018

Simulou-se no Matlab® cada combinação apresentada na Tabela 2 Os resultados que devem ser obtidos são: tensão no no nó 3 (V3V), tensão no nó 4 (V4V), diferença de tensão entre os nós 3 e 4 (dV34) e a corrente injetada (IT). Na Tabela 3, tem-se o resumo dos resultados possíveis de obter. Nas combinações em que o sistema foi impossível de ser definido, utilizando o método Loop Flow, descreveu-se como não convergência.

TABELA 3. Caracterização do sistema, utilizando método Loop Flow, para cada combinação de defasagem.

\begin{tabular}{|c|c|c|c|c|c|}
\hline $\begin{array}{l}E 1 \\
(\mathrm{~V})\end{array}$ & $\begin{array}{l}E 2 \\
(\mathrm{~V})\end{array}$ & $\begin{array}{l}V 3 V \\
(\mathrm{~V})\end{array}$ & $\begin{array}{l}\mathrm{V} 4 \mathrm{~V} \\
(\mathrm{~V})\end{array}$ & $\begin{array}{c}d V 34 V \\
(\mathrm{~V})\end{array}$ & $\begin{array}{l}I T \\
\text { (A) }\end{array}$ \\
\hline $7200<-120^{\circ}$ & $7560<-120^{\circ}$ & $6970,48<-1,33^{\circ}$ & $6970,48<-1,33^{\circ}$ & $919,2 \mu$ & $36,88<-14,65^{\circ}$ \\
\hline $7200<-120^{\circ}$ & $7560<-120^{\circ}$ & \multicolumn{4}{|c|}{ Sistema não converge! } \\
\hline $7200<-120^{\circ}$ & $7560<-120^{\circ}$ & \multicolumn{4}{|c|}{ Sistema não converge! } \\
\hline $7200<-120^{\circ}$ & $7560<-120^{\circ}$ & \multicolumn{4}{|c|}{ Sistema não converge! } \\
\hline $7200<-120^{\circ}$ & $7560<-120^{\circ}$ & $6970,46<118,67^{\circ}$ & $6970,46<118,67^{\circ}$ & $919,2 \mu$ & $36,88<105,35^{\circ}$ \\
\hline $7200<-120^{\circ}$ & $7560<-120^{\circ}$ & \multicolumn{4}{|c|}{ Sistema não converge! } \\
\hline $7200<-120^{\circ}$ & $7560<-120^{\circ}$ & \multicolumn{4}{|c|}{ Sistema não converge! } \\
\hline $7200<-120^{\circ}$ & $7560<-120^{\circ}$ & \multicolumn{4}{|c|}{ Sistema não converge! } \\
\hline $7200<-120^{\circ}$ & $7560<-120^{\circ}$ & $6970,43<-121,33^{\circ}$ & $6970,43<-121,33^{\circ}$ & $919,2 \mu$ & $36,88<-134,64^{\circ}$ \\
\hline
\end{tabular}

Fonte: Autoria própria, 2018.

De acordo com simulações, pode-se afirmar que o método do Loop Flow, analisado para o circuito da Figura 1, conforme apresentado por Kersting, não se aplica a casos em que as fontes de tensão apresentam desequilíbrios. Para fontes com defasagens, só ocorreu a convergência quando as defasagens de E1 e E2 eram iguais.

Uma adaptação ao método foi realizada, a fim de que o mesmo tornasse compatível com sistemas desbalanceados. Neste, utilizou-se o circuito conforme a Figura 7. 


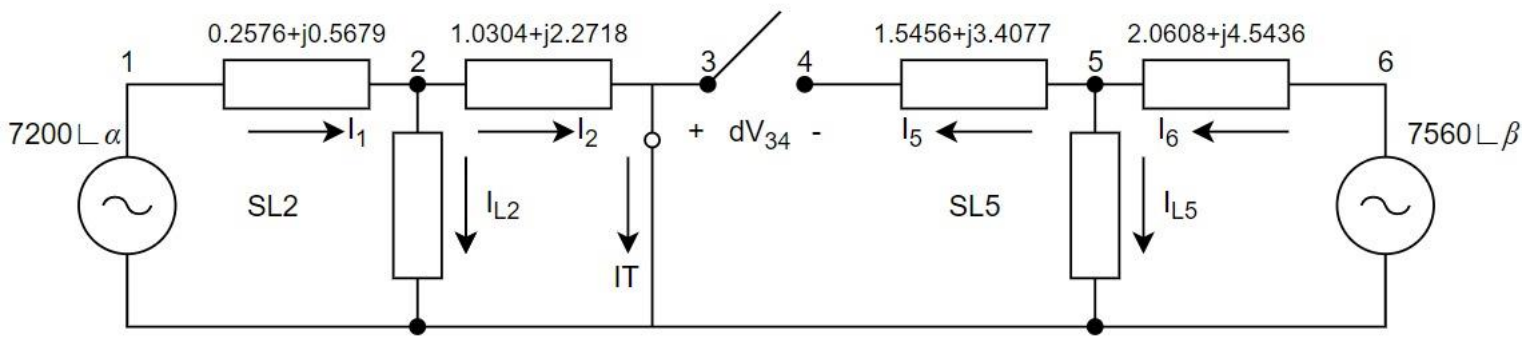

FIGURA 7. Adaptação método Loop Flow. (Fonte: Adaptado KERSTING, 2017).

A partir do circuito da Figura 7 desenvolveu-se um novo script em Matlab®. O mesmo é diferente do script da Tabela 1, pois considera apenas uma fonte de injeção de corrente, o que acarreta em mudanças na análise do circuito. Na Tabela 4, demonstra-se a modificação no script anteriormente apresentado. Como houve modificação apenas no passo 6, somente o mesmo foi reescrito e representado na Tabela 4, o mesmo foi reescrito, pois a corrente IT terá influência apenas no lado esquerdo do circuito. Com exceção do passo 6, todos os demais passos são iguais ao do script do método Loop Flow. O script completo, encontra-se no Anexo A deste trabalho.

TABela 4. Adaptação no script do método Loop Flow.

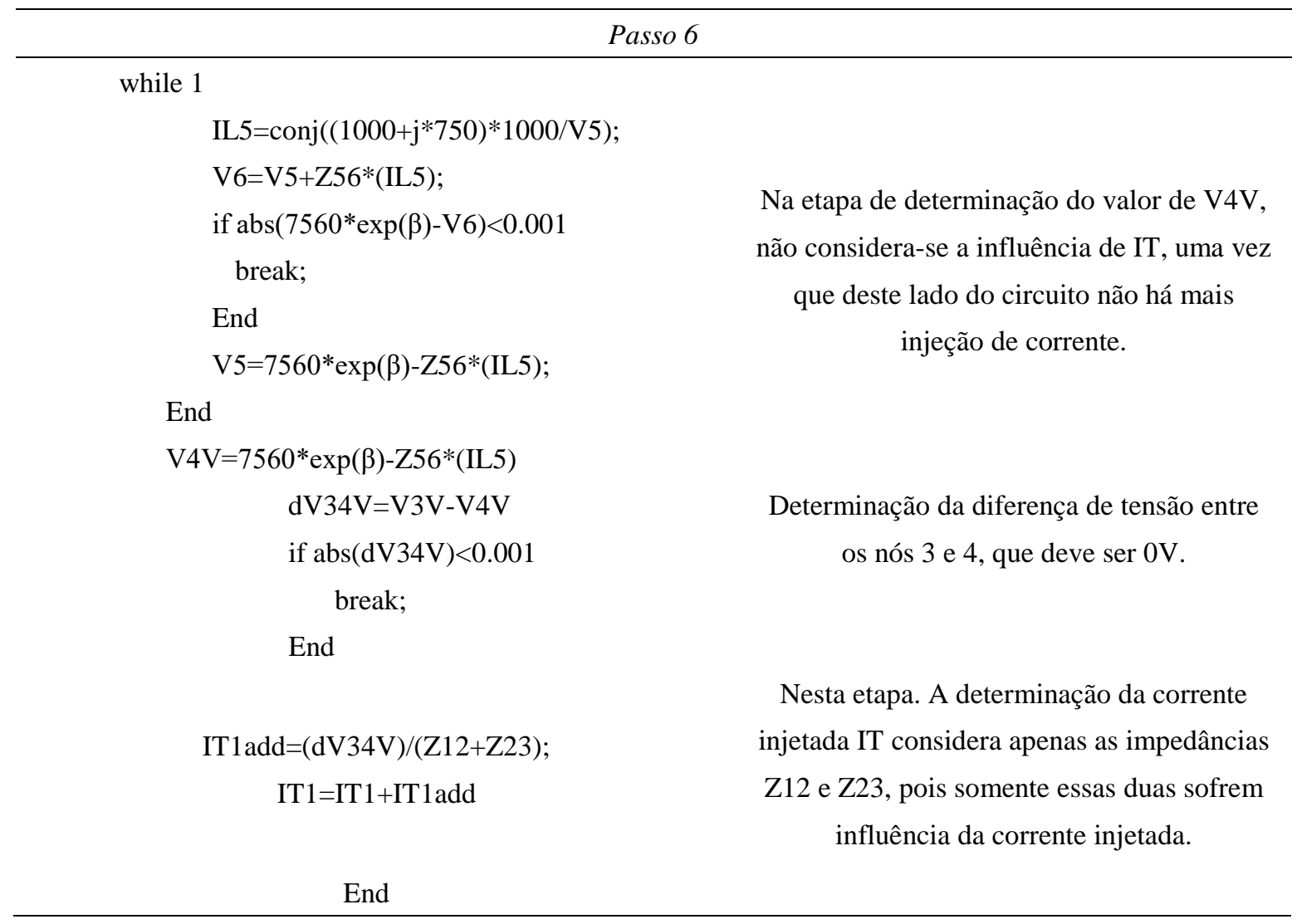

Fonte: Autoria própria (2018).

Com o novo script simulou-se as combinações das defasagens, conforme a Tabela 2. Os resultados obtidos estão demonstrados na Tabela 5. 
TABELA 5. Resultados obtidos com a simulação, utilizando o script adaptado.

\begin{tabular}{|c|c|c|c|c|c|c|}
\hline $\begin{array}{l}E 1 \\
(\mathrm{~V})\end{array}$ & $\begin{array}{l}E 2 \\
(\mathrm{~V})\end{array}$ & $\begin{array}{l}V 3 V \\
(\mathrm{~V})\end{array}$ & $\begin{array}{l}V 4 \mathrm{~V} \\
(\mathrm{~V})\end{array}$ & $\begin{array}{c}d V 34 V \\
(\mathrm{~V})\end{array}$ & $\begin{array}{l}I T \\
\text { (A) }\end{array}$ & $I t^{1}$ \\
\hline $7200<$ & $7560<\quad 0^{\circ}$ & $6734,89<-3,37^{\circ}$ & $6734,89<-3,37^{\circ}$ & $238,83 \mu$ & $144,57<-18,99^{\circ}$ & 4 \\
\hline $7200<$ & $7560<120^{\circ}$ & $6734,95<116,62^{\circ}$ & $6734,95<116,62^{\circ}$ & $163,55 \mu$ & $3861,1<-96,22^{\circ}$ & 5 \\
\hline $7200<0^{\circ}$ & $7560<-120^{\circ}$ & $6734,88<-123,37^{\circ}$ & $6734,88<-123,37^{\circ}$ & $191,15 \mu$ & $3968,43<-37,63^{\circ}$ & 5 \\
\hline $7200<120^{\circ}$ & $7560<\quad 0^{\circ}$ & $6734,89<-3,37^{\circ}$ & $6734,89<-3,37^{\circ}$ & $191,14 \mu$ & $3968,47<82,37^{\circ}$ & 5 \\
\hline $7200<120^{\circ}$ & $7560<120^{\circ}$ & $6734,95<116,62^{\circ}$ & $6734,95<116,62^{\circ}$ & $238,83 \mu$ & $144,56<101,01^{\circ}$ & 4 \\
\hline $7200<120^{\circ}$ & $7560<-120^{\circ}$ & $6734,88<-123,37^{\circ}$ & $6734,88<-123,37^{\circ}$ & $163,55 \mu$ & $3861,1<23,78^{\circ}$ & 5 \\
\hline $7200<-120^{\circ}$ & $7560<$ & $6734,89<-3,37^{\circ}$ & $6734,89<-3,37^{\circ}$ & $163,54 \mu$ & $3861,1<143,78^{\circ}$ & 5 \\
\hline $7200<-120^{\circ}$ & $7560<120^{\circ}$ & $6734,91<116,62^{\circ}$ & $6734,91<116,62^{\circ}$ & $191,15 \mu$ & $3968,45<-157,63^{\circ}$ & 5 \\
\hline $7200<-120^{\circ}$ & $7560<-120^{\circ}$ & $6734,88<-123,37^{\circ}$ & $6734,88<-123,37^{\circ}$ & $238,83 \mu$ & $144,56<-138,99^{\circ}$ & 4 \\
\hline
\end{tabular}

Fonte: Autoria própria (2018).

${ }^{1}$ It: Iterações.

Conforme apresentado, com a adaptação do método Loop Flow, pode-se obter convergência para as variadas combinações de ângulos de defasagens para as fontes de tensão.

\subsection{Validação do Método}

O método pode ser validado com base em mismatches ou resíduos de corrente ou de tensão, com base nas leis de Kirchhoff. Considerando, por exemplo, o circuito da figura 7 e E1 e E2 no primeiro caso: $\mathrm{E} 1=7200<0^{\circ}$ e E2 $=7560<0^{\circ}$. Pode-se ver que a fonte de corrente calculada para fechar a malha entre as duas fontes foi de 144,57<-18,99 . Calcula-se então usando o script do Apêndice as correntes do circuito e as tensões nas fontes, chegando-se aos valores: $E 1$ calc $=7.2000 \mathrm{e}+03+1.8048 \mathrm{e}-04 \mathrm{i}$ e E2calc=7.5600e+03 - 3.8681e-05i. Assim o erro nas tensões E1 e E2 que o método obteve foram de 3.2549e-06 \% para E1 e de 8.6745e-06 \% para E2. Portanto, como o erro é pequeno, o método é validado. A fonte de corrente iguala o potencial das duas malhas e força a corrente $\mathrm{I}_{2}=\mathrm{IT}$ e $\mathrm{I}_{5}=0$ na rede não-linear com cargas de potência constante.

\section{CONSIDERAÇÕES FINAIS}

O objetivo principal deste trabalho é analisar o método Loop Flow para situações não apresentadas pelo autor do método, como por exemplo o uso de fontes de tensão com defasagens.

O equacionamento fundamental do método Loop Flow, bem como seu algoritmo, foram apresentados e detalhados. Os resultados obtidos, através de simulações no Matlab®, demonstraram a não eficácia do método, para uso em um sistema com fontes de tensão defasadas, nestes casos o método não convergiu.

A fim de obter-se uma análise estendida, adaptou-se o método Loop Flow, considerando um circuito modificado, com apenas uma corrente injetada em um único lado do circuito. A adaptação do método mostrouse positiva, pois o mesmo convergiu para as combinações de defasagens analisadas.

Métodos alternativos para análise de sistemas de distribuição, são de extrema importância, uma vez que na análise de fluxo de potência há um maior foco em métodos para sistemas de transmissão, os quais não apresentam boa aplicabilidade em sistemas de transmissão.

Este trabalho pôde ser concluído com êxito, uma vez que seu objetivo de analisar o método Loop Flow, foi atendido, demonstrando os valores para os quais o sistema não apresentava convergência. 
APÊNDICE A1 - SCRIPT DO MÉTODO ADAPTADO

$\mathrm{E} 1=7200 * \exp (\alpha)$;

$\mathrm{E} 2=7560 * \exp (\beta)$;

$\mathrm{Z} 12=0.2576+\mathrm{j} * 0.5679$;

$\mathrm{Z} 23=1.0304+\mathrm{j} * 2.2718$;

$\mathrm{Z} 45=1.5456+\mathrm{j} * 3.4077$;

$\mathrm{Z} 56=2.0608+\mathrm{j} * 4.5436$;

IT1=138.9;

IT2=-138.9;

$\mathrm{V} 3 \mathrm{I}=-(\mathrm{Z} 12+\mathrm{Z} 23) * \mathrm{IT} 1$;

$\mathrm{V} 4 \mathrm{I}=(\mathrm{Z} 56+\mathrm{Z} 45) * \mathrm{IT} 2$;

dV34I=V3I-V4I;

Zth=dV34I/IT1;

\%Primeira ladder iterative technique para determinar V3V

$\mathrm{V} 2=7200 * \exp (\alpha)$

while 1

IL2 $=\operatorname{conj}((1500+\mathrm{j} * 1250) * 1000 / \mathrm{V} 2)$;

$\mathrm{V} 1=\mathrm{V} 2+\mathrm{Z} 12 * \mathrm{IL} 2$

if $\operatorname{abs}(7200 * \exp (\alpha)-\mathrm{V} 1)<0.001$

break;

end

$\mathrm{V} 2=7200 * \exp (\alpha)-\mathrm{Z} 12 * \operatorname{IL} 2 ;$

end

V3V=7200*exp( $\alpha)-Z 12 * I L 2 ;$

$\mathrm{V} 5=7560 * \exp (\beta)$;

while 1

IL5=conj $((1000+j * 750) * 1000 / V 5)$;

$\mathrm{V} 6=\mathrm{V} 5+\mathrm{Z} 56 * \mathrm{IL} 5$;

if $\operatorname{abs}\left(7560^{*} \exp (\beta)-\mathrm{V} 6\right)<0.001$

break;

end

V5 $=7560 * \exp (\beta)-Z 56 *$ IL5;

end

$\mathrm{V} 4 \mathrm{~V}=7560 * \exp (\beta)-Z 56 *$ IL5;

IT1 $=0$;

$\mathrm{dV} 34 \mathrm{~V}=\mathrm{V} 3 \mathrm{~V}-\mathrm{V} 4 \mathrm{~V}$;

IT2=0;

Pause

\% Todo o processo é repetido agora com ITadd adicionado, ou seja, com o novo $\%$ valor de IT 
while 1

$\mathrm{V} 2=7200 * \exp (\alpha)$;

while 1

IL2=conj $((1500+j * 1250) * 1000 / \mathrm{V} 2)$;

$\mathrm{V} 1=\mathrm{V} 2+\mathrm{Z} 12 *(\mathrm{IL} 2+\mathrm{IT} 1)+\mathrm{Z} 23 * \mathrm{IT} 1$;

if $\operatorname{abs}\left(7200^{*} \exp (\alpha)-\mathrm{V} 1\right)<0.001$

break;

end

$\mathrm{V} 2=7200 * \exp (\alpha)-\mathrm{Z} 12 *(\mathrm{IL} 2+\mathrm{IT} 1)-\mathrm{Z} 23 * \mathrm{IT} 1$;

end

$\mathrm{V} 3 \mathrm{~V}=7200 * \exp (\alpha)-\mathrm{Z} 12 *(\mathrm{IL} 2+\mathrm{IT} 1)-\mathrm{Z} 23 * \mathrm{IT} 1$

$\mathrm{V} 5=7560 * \exp (\beta)$;

while 1

IL5=conj $((1000+j * 750) * 1000 / \mathrm{V} 5)$;

V6=V5+Z56*(IL5);

if $\operatorname{abs}(7560 * \exp (\beta)-\mathrm{V} 6)<0.001$

break;

end

V5=7560*exp( $\beta)-Z 56 *(I L 5) ;$

end

$\mathrm{V} 4 \mathrm{~V}=7560 * \exp (\beta)-Z 56 *($ IL5)

$\mathrm{dV} 34 \mathrm{~V}=\mathrm{V} 3 \mathrm{~V}-\mathrm{V} 4 \mathrm{~V}$

$\operatorname{abs}(\mathrm{dV} 34 \mathrm{~V})$;

pause

if $\operatorname{abs}(\mathrm{dV} 34 \mathrm{~V})<0.001$

break;

end

IT1add $=(\mathrm{dV} 34 \mathrm{~V}) /(\mathrm{Z} 12+\mathrm{Z} 23)$;

$\mathrm{IT} 1=\mathrm{IT} 1+\mathrm{IT} 1 \mathrm{add}$

end

\section{REFERÊNCIAS}

[1] SILVA, David Maykon Krepsky. Fluxo de Potência para Sistemas de Distribuição Considerando Redução de Barras. 2016. 73 p. TCC (Graduação) - Curso de Engenharia Elétrica, Departamento de Engenharia Elétrica, Universidade Estadual de Londrina, Londrina, 2016.

[2] MOURA, A. P.; MOURA, A. A. F.; ROCHA, E. P. Análise de Fluxo de Carga em Sistemas de Potência. Artliber. 2018.

[3] KERSTING, William H. Distribution System: Modeling and Analysis. 4. ed. Boca Raton: CRC Press, 2017. $526 \mathrm{p}$.

[4] VALDIR, A. P. Desempenho de um algoritmo backward-forward sweep de cálculo de fluxo de potência. FEIS. 2006.

[5] CARVALHO, Marcus Rodrigo. Estudo Comparativo de Fluxo de Potência para Sistemas de Distribuição Radiais. 2006. 104 f. Dissertação (Mestrado) - Curso de Engenharia Elétrica, Escola de Engenharia de São Carlos, Universidade de São Paulo, São Carlos, 2006. 
[6] HAQUE, M. H. Efficient Load Flow Method for Distribution Systems with Radial or Mesh Configuration. IEE Proceedings - Generation, Transmission and Distribution.Vol. 143, n 1, 1996.

[7] SIVANAGARAJUL, S.; VISWANATHA RAO, J. and GIRIDHAR, M. A Loop Based Load Flow Method for Weakly Meshed Distribution Network. ARPN Journal of Engineering and Applied Sciences. Vol. 3, $\mathrm{n}^{\circ}$ 4, 2008.

[8] HUSAIN, T.;MUQUEEM K.; ANSARI, M. M. Load Flow Analysis of Radial and Mesh Distribution System Using ZIP Model. International Conference on Global Trends in Signal Processing, Information Computing and Communication (ICGTSPICC). 2016. 